\title{
Maximizing acquisition efficiency through simultaneous source technology
}

Ray Abma and Mark Foster, BP

Simultaneous source acquisition can significantly improve the efficiency of seismic data acquisition (Howe et al 2008, Howe et al 2009, Bouska et al 2008). BP has developed seismic acquisition technologies like $\mathrm{DS}^{3}, \mathrm{ISS}^{\mathrm{TM}}$ and ISSN ${ }^{\mathrm{TM}}$ which can efficiently deliver high-quality data in desert terrains, such that land 3D can be acquired for exploration and development purposes at costs approaching conventional 3D marine towed-streamer surveys. In addition to the very high productivity rates, simultaneous source technology enables the economic acquisition of well sampled, high fold wide azimuth surveys which delivers the observed improvement in data quality, and together with new noise separation techniques, like BP's SSI technique (Abma et al 2010), these methods are being used for development quality 3D surveys where high quality pre-stack data and wide azimuth attributes are required.

We will show examples of simultaneous source acquisition from BP's surveys to date, and review the experience gained as well as looking at future opportunities and challenges. To summarize our experience, it appears that we can acquire seismic surveys with simultaneous sources in a manner that is faster, cheaper, and better than conventional surveys. 